\title{
Metástases Cerebrais
}

\author{
Adrialdo José Santos ${ }^{1}$ \\ Clélia Maria Ribeiro Franco ${ }^{1}$ \\ Lia Raquel Rodrigues Borges ${ }^{1}$ \\ Suzana Maria Fleury Malheiros ${ }^{2}$ \\ Alberto Alain Gabbai ${ }^{3}$
}

\begin{abstract}
RESUMO
As metástases cerebrais ocorrem comumente em pacientes com câncer. A frequiência dessa complicação está aumentando em decorrência do tratamento mais agressivo do câncer, bem como do uso de métodos de neuroimagem que permitem um diagnóstico mais preciso. Com o melhor controle dos tumores primários, permitido pelos tratamentos atuais, a importância do tratamento dessas metástases é óbvia. Os principais objetivos do tratamento são melhorar os déficits neurológicos e aumentar a expectativa e a qualidade de vida. Os autores revisam as manifestações clínicas, o diagnóstico e as principais opções para o tratamento das metástases cerebrais.
\end{abstract}

Unitermos: Metástases intracranianas, cirurgia, radioterapia, tratamento clínico.

\section{Introdução}

O tempo e a qualidade de vida dos pacientes oncológicos têm aumentado sensivelmente com os tratamentos atuais. Contudo, o aumento na sobrevida tem algumas conseqüências, como, por exemplo, o fato de as complicações metastáticas serem cada vez mais freqüentes. Neste contexto, as metástases intracranianas ainda são consideradas a complicação neurológica mais freqüente e temível, pois são responsáveis por sintomas incapacitantes ou por morte precoce e, muitas vezes, implicam a desistência do tratamento sistêmico por alguns médicos. As metástases intracranianas ocorrem mais comumente nas fases avançadas do câncer, mas podem ser a primeira manifestação de um tumor primário desconhecido ${ }^{1,2}$. Seu diagnóstico precoce é importante para promover não apenas maior sobrevida, mas também para melhorar a qualidade de vida dos pacientes.

Estima-se que cerca de $20 \%$ a $25 \%$ dos pacientes com câncer desenvolvam metástases cerebrais. Estudos baseados em necropsias estimam a ocorrência das metástases intracranianas (MIC) em até $25 \%$ dos pacientes que morreram de câncer ${ }^{1,3}$. As MIC podem localizar-se no parênquima (hemisférios cerebrais, cerebelo, tronco encefálico) ou nas meninges (dura-máter e leptomeninges). Nesta revisão, serão discutidas as metástases intraparenquimatosas, que chamaremos de metástases cerebrais, por ser este o termo mais consagrado pelo uso. As metástases cerebrais representam $2 / 3$ das MIC, podendo ser únicas ou múltiplas. Alguns tumores, como o melanoma, geralmente produzem metástases múltiplas, enquanto outros, como o carcinoma de

\footnotetext{
Pós-graduandos da Disciplina de Neurologia da Escola Paulista de Medicina - Unifesp.

Responsável pelo Setor de Neuro-oncologia da Escola Paulista de Medicina - Unifesp.

Professor Titular e Chefe da Disciplina de Neurologia da Escola Paulista de Medicina - Unifesp.
} 
mama, mais freqüentemente originam uma única lesão. O termo metástase cerebral solitária implica a ausência de qualquer outra lesão metastática conhecida, enquanto o termo metástase cerebral única indica que há uma única lesão cerebral independentemente da existência de metástases sistêmicas ${ }^{4}$.

Os tumores primários que mais freqüentemente evoluem com metástases para o sistema nervoso central (SNC) em adultos são o carcinoma broncogênico (principalmente o carcinoma de pequenas células e o adenocarcinoma), o câncer de mama, o carcinoma renal, o melanoma e as neoplasias malignas do trato gastrointestinal, embora até $10 \%$ das metástases cerebrais tenham origem primária desconhecida $^{2,3}$. Outros tumores como o carcinoma de próstata, ovário, testículo e o linfoma de Hodgkin raramente apresentam metástases cerebrais ${ }^{3}$. Nos pacientes com idade inferior a 21 anos, as metástases originam-se principalmente dos sarcomas (sarcoma osteogênico, rabdomiossarcoma e sarcoma de Ewing) e dos tumores de células germinativas ${ }^{1,3}$.

A maioria das metástases cerebrais ocorre por disseminação hematogênica, principalmente pela circulação arterial e, em alguns casos, pode ocorrer pelo sistema venoso por meio do plexo venoso vertebral (plexo de Batson) ${ }^{1,5}$. A distribuição das metástases é aproximadamente proporcional ao fluxo sangüíneo cerebral e isto é observado pelo predomínio das lesões nos lobos frontal e parietal ${ }^{5}$. De modo geral, aproximadamente $80 \%$ das metástases cerebrais localizam-se nos hemisférios cerebrais, $15 \%$ no cerebelo e 5\% no tronco cerebral. A fossa posterior é acometida preferencialmente por lesões originadas dos tumores pélvicos ${ }^{1,5}$.

As metástases localizam-se preferencialmente na região de transição córtico-subcortical, que é uma interface bem vascularizada, denominada "zona de fronteira" (watershed) ou de circulação terminal. Isto sugere que microêmbolos se estabeleçam nos capilares distais das artérias superficiais ${ }^{6}$.

\section{Manifestações clínicas}

Os tumores cerebrais metastáticos manifestamse com os mesmos sinais clínicos dos tumores intracranianos primários, distinguindo-se destes por sua evolução mais rápida, geralmente subaguda, em dias ou poucas semanas. Geralmente, essa evolução clínica mais rápida deve-se, entre outros fatores, ao intenso edema perilesional ${ }^{3}$. Os sintomas progressivos de aumento da pressão intracraniana (cefaléia, alteração do nível de consciência), alterações de comportamento, sinais neurológicos focais e crises epilépticas são as manifestações clínicas habituais dos processos metastáticos cerebrais. Cerca de 10\% dos casos têm apresentação brusca, apoplética, secundária à hemorragia intratumoral, constituindo as chamadas "síndromes pseudovasculares" 4 . A hemorragia espontânea pode ocorrer em qualquer tipo de neoplasia metastática, sendo mais freqüente no melanoma, no carcinoma renal e no coriocarcinoma (os quais apresentam invasão vascular) ${ }^{3}$. Uma complicação precoce das metástases cerebelares é a hidrocefalia obstrutiva que, se não tratada a tempo, é seguida de rápida deterioração clínica e óbito. Alterações comportamentais são observadas em até $30 \%$ dos pacientes ${ }^{4}$.

\section{Diagnóstico}

A maioria das metástases cerebrais aparece como lesões arredondadas, com realce difuso ou anelar, tipicamente circundadas por intenso edema perilesional, o qual não guarda proporção com o tamanho da lesão. O efeito expansivo também é variável e, nos casos de apresentação aguda com hemorragia intratumoral, esta pode dificultar o diagnóstico de neoplasia subjacente ${ }^{3}$. Os principais métodos de neuroimagem para o diagnóstico de metástases cerebrais são a tomografia computadorizada e a ressonância magnética contrastadas. Têm sido descritas variações de técnica, como, por exemplo, o uso de contraste em doses maiores do que o habitual, bem como o aumento do intervalo de tempo entre a injeção do contraste e a aquisição da imagem, com o objetivo de aumentar a sensibilidade diagnóstica desses métodos. A ressonância magnética com gadolínio é considerada o método de escolha para avaliação das lesões suspeitas, sendo especialmente útil nos pacientes com imagem tomográfica duvidosa ou em localização que não é bem visibilizada pela tomografia (por exemplo, fossa posterior) ${ }^{7,8}$. Além disso, a ressonância pode fornecer melhores informações com relação à localização anatômica, à diferenciação e ao número de lesões e também evita o risco de anafilaxia pelo contraste iodado. Entretanto, em algumas situações como nos casos de hemorragia aguda ou envolvimento metastático da calota craniana, a tomografia computadorizada tem melhor resolução ${ }^{3,4,7,8}$.

$\mathrm{Na}$ maioria dos pacientes com história conhecida de câncer, a certeza de que uma lesão cerebral única, apresentando realce, corresponda a uma metástase gira em torno de $90 \%{ }^{3}$. Contudo, há exceções e, portanto, uma lesão não deve ser classificada como 
metastática sem a definitiva comprovação histopatológica ${ }^{3,4}$. Também vale ressaltar que lesões de natureza diversa podem ter aspecto radiológico sugestivo de metástase. Como exemplos podem ser citados os processos inflamatórios e infecciosos (granulomas, abscessos), vasculares (hemorragias em reabsorção, infartos) e neoplasias cerebrais primárias, que podem ocorrer mesmo nos pacientes com câncer sistêmico conhecido ${ }^{3,4,8}$.

Por outro lado, pacientes sem diagnóstico prévio de câncer podem apresentar sintomas neurológicos e achados de neuroimagem sugestivos de metástases. Esses casos constituem um desafio para o diagnóstico, devendo ser cuidadosamente abordados. $\mathrm{O}$ exame clínico minucioso pode detectar tumores primários do reto, dos testículos, da próstata, da mama, dos linfonodos e da pele (melanoma). Como muitas das metástases cerebrais originam-se no pulmão e como também é freqüente o acometimento concomitante desse órgão na maioria das neoplasias, deve-se dar especial atenção ao tórax. Neste caso, recomenda-se a realização de radiografia de tórax e citologia do escarro, estendendo-se a investigação com tomografia, ressonância, broncospia com lavado e biópsia nos casos suspeitos ${ }^{4}$. O mapeamento ósseo também pode detectar lesões metastáticas, eventualmente acessíveis para biópsia. A tomografia de abdome e pelve pode ser útil para o diagnóstico de carcinoma renal e de outras neoplasias abdominais. Exames relativamente simples, como a pesquisa de sangue oculto nas fezes, podem fornecer informações relevantes e direcionar a investigação clínica. Marcadores bioquímicos, como CEA, alfa-fetoproteína e CA 125 , podem ser úteis na pesquisa de tumores embrionários. Nas mulheres, recomenda-se especial atenção à possibilidade de carcinoma de mama, indicando-se a realização de mamografia. Se após extensa investigação não for encontrado o tumor primário e se a lesão cerebral for acessível à cirurgia, indica-se a sua exérese para diagnóstico e eventual tratamento ${ }^{4}$. No caso de lesões múltiplas ou quando a lesão única for de difícil acesso, a biópsia por estereotaxia pode ser mais adequada, embora exista o risco de disseminação tumoral ao longo do trajeto da agulha utilizada nesse procedimento ${ }^{8,9}$.

\section{Tratamento}

A literatura disponível sobre a história natural das metástases cerebrais sugere uma sobrevida mediana em torno de 7 semanas, quando as lesões sintomáticas não são tratadas ${ }^{4}$, com óbito geralmente decorrente de aumento da pressão intracraniana e conseqüente herniação cerebral produzindo compressão do tronco, ou de intercorrências clínicas (distúrbios metabólicos e infecciosos). Todas as evidências disponíveis, até o momento, indicam que a sobrevida é maior e com melhor qualidade de vida se as metástases cerebrais forem tratadas ${ }^{3,4,8}$.

O tratamento das metástases cerebrais compreende duas etapas fundamentais: o tratamento sintomático e o específico. O primeiro visa à estabilização do paciente crítico e inclui o controle da pressão intracraniana (com uso de corticosteróides e/ou de agentes hiperosmolares) e o tratamento de eventuais crises epilépticas, dos distúrbios metabólico e infeccioso, bem como dos estados de hipercoagulabilidade que podem acompanhar alguns tipos de câncer ${ }^{1,3,10,11}$.

Com o paciente em situação estável, o tratamento específico dependerá do número e da localização das lesões, do diagnóstico e do estadiamento do tumor primário, bem como do comportamento biológico da neoplasia primária.

\section{Tratamento sintomático}

Os corticosteróides constituem o primeiro tratamento para os pacientes com metástases cerebrais desde $1960^{4,8}$. A melhora clínica é usualmente evidente dentro de 24 a 48 horas após o início da corticoterapia e dá-se preferência ao uso da dexametasona na dose de $16 \mathrm{mg} / \mathrm{dia}$, em doses fracionadas, ou mesmo doses maiores, quando não há resposta significativa em 48 horas. Embora se acredite que essa melhora seja decorrente da redução do edema cerebral, o exato mecanismo de ação ainda é desconhecido. Tem sido sugerido que essa melhora esteja relacionada com alterações no metabolismo celular $^{8,12}$. O tratamento específico deve ser então instituído, com redução progressiva do corticosteróide até a mínima dose possível. Os efeitos colaterais do uso de corticosteróides incluem o risco de desenvolvimento ou agudização de úlceras pépticas, alterações metabólicas (por exemplo, hiperglicemia), miopatia, hipertensão arterial, síndrome de Cushing, distúrbios do sono, ganho ponderal, psicose, tremores, bem como infecções oportunistas. Também é conhecida a potencial interação da dexametasona com a fenitoína, recomendando-se a monitorização dos níveis séricos de fenitoína nos pacientes que estejam usando essa associação ${ }^{8}$.

Os pacientes com metástases cerebrais podem requerer o uso de drogas antiepilépticas (DAE) para tratamento ou para profilaxia de crises epilépticas. 
Alguns autores sugerem que os pacientes com metástases de melanoma têm maior risco de crises, devendo receber DAE profilaticamente ${ }^{8}$. Entretanto, o uso dessas drogas nos pacientes que não apresentaram crises ou que ainda não foram submetidos à cirurgia é muito controverso. Quando o uso é indicado, a fenitoína é a primeira droga de escolha devido à disponibilidade de uso endovenoso e seu menor efeito sedativo. Contudo, há relatos de síndrome de StevensJohnson após a radioterapia, em pacientes que usavam fenitoína ${ }^{8,13}$. Já foi demonstrado in vitro que a fenitoína pode estimular a proliferação linfocitária e, por outro lado, a radioterapia pode diminuir a atividade dos linfócitos $\mathrm{T}$ supressores. Deste modo, esses dois fatores poderiam explicar o efeito sinérgico da radioterapia e da fenitoína, aumentando a incidência da síndrome de Stevens-Johnson ${ }^{8}$.

Muitos pacientes com câncer apresentam distúrbios de coagulação e a complicação mais freqüente é a tromboflebite, com ou sem embolia pulmonar ${ }^{10}$, tornando necessário, nestes casos, o tratamento profilático e, eventualmente, a anticoagulação. Vale ressaltar que a incidência de hemorragia intratumoral não é maior nos pacientes com tumores cerebrais primários ou metastáticos em uso de anticoagulantes do que naqueles que não os estejam usando ${ }^{10}$.

\section{Tratamento específico}

As opções de tratamento para os pacientes com metástases cerebrais incluem o uso de quimioterapia, radioterapia de encéfalo total ou localizada, cirurgia convencional e radiocirurgia. Para os pacientes com metástase cerebral única, a ressecção cirúrgica permanece como importante opção terapêutica ${ }^{4,8}$. As vantagens da cirurgia incluem a possibilidade de estabelecer ou confirmar o diagnóstico, melhorar os déficits neurológicos e permitir o controle local do tumor ${ }^{4,8}$. Além disto, com os avanços cirúrgicos ocorridos nos últimos anos, tem-se observado considerável redução da morbidade. Exemplos destes avanços incluem o uso de métodos que permitem melhor localização do tumor como ultrassonografia, tomografia de crânio e ressonância magnética intraoperatórios e também a utilização de exames funcionais que possibilitam a identificação de áreas eloqüentes como o córtex motor primário, diminuindo assim as seqüelas neurológicas ${ }^{8}$. O tratamento clássico da metástase cerebral única consiste na ressecção cirúrgica seguida de radioterapia, proporcionando uma sobrevida mediana de 40 semanas, a qual é significativamente maior que as 15 semanas obtidas com radioterapia como tratamento único ${ }^{8,14,15}$. O aumento da sobrevida observado nos pacientes com metástase única submetidos a tratamento cirúrgico tem entusiasmado alguns neurocirurgiões a considerar a possibilidade de cirurgia nos pacientes com metástases múltiplas, o que é particularmente útil no caso de lesões radiorresistentes como as do melanoma e dos carcinomas de cólon e $\operatorname{rim}^{4,8}$. De modo geral, preconiza-se a cirurgia para pacientes com até 3 lesões ${ }^{4}$.

A radioterapia externa convencional é indicada classicamente para os pacientes com múltiplas metástases ou com metástase única associada a doença sistêmica disseminada. $\mathrm{O}$ esquema padronizado pela maioria dos serviços consiste em radiação externa de megavoltagem com dose total variando de 30 a $45 \mathrm{~Gy}$. Têm sido descritas variações de dose e de fracionamento, mas, excetuando-se os esquemas ultrarápidos de altas doses, os quais são associados com maior morbidade e recidiva precoce, ainda não foram demonstradas diferenças significativas nestes esquemas $^{4,8,16}$. A sobrevida mediana proporcionada pela radioterapia é de 3 a 6 meses e cerca de 10 a $15 \%$ dos pacientes sobrevivem além do $1^{\circ}$ ano $^{4}$. Entretanto, vale ressaltar que, como a maioria dos pacientes com metástases cerebrais morre em função da progressão sistêmica da doença, a sobrevida não deve ser o único parâmetro considerado para a avaliação da eficácia da radioterapia, devendo ser analisados outros fatores, como controle local da doença e tempo livre de sintomas neurológicos ${ }^{4,8,16-18}$.

A radioterapia localizada com fonte externa (radiocirurgia extereotáxica ou gamma-knife) ou com uso de implantes (braquiterapia) consiste numa opção terapêutica que vem ganhando força nos últimos anos ${ }^{4}$. Estas modalidades de radioterapia objetivam a utilização de uma dose focal de radiação, concentrando-a no alvo e diminuindo os efeitos nos tecidos adjacentes. É uma opção para as lesões inacessíveis cirurgicamente e as metástases são o alvo preferencial para esta modalidade de tratamento porque têm formato aproximadamente esférico e geralmente não invadem os tecidos vizinhos. A radiocirurgia é geralmente bem tolerada, embora sejam descritos efeitos colaterais como náuseas e vômitos em pacientes com tumores na região do IV ventrículo ${ }^{4,8}$. Ainda não há consenso sobre a eficácia da radiocirurgia em relação à cirurgia convencional, sendo recomendada para pacientes com lesões de difícil acesso e de pequeno tamanho. Os critérios que indicam melhor prognóstico com a radiocirurgia são a presença de lesão única, índice de Karnofsky maior que 70 e ausência de doença sistêmica disseminada ${ }^{19,20-24}$. 
A braquiterapia consiste no implante de fontes de radiação que podem ser de alta e baixa dosagem de Iodo 125. Os de baixa dosagem são implantados sob craniotomia enquanto os de alta são colocados temporariamente por meio de extereotaxia ${ }^{8}$. Até o momento, a braquiterapia tem lugar limitado no tratamento das metástases cerebrais, ficando reservada para os pacientes que esgotaram outras possibilidades terapêuticas ${ }^{8}$.

O comportamento biológico da neoplasia também deve ser considerado, pois alguns tumores são mais radiossensíveis que outros, como descrito por Cairncross et al. ${ }^{16}$. Segundo estes autores, as metástases cerebrais dos carcinomas de mama e pulmão apresentam boas respostas clínica e tomográfica à radioterapia. Já os pacientes com metástases de melanoma, carcinoma renal e de cólon, embora tenham uma aparente melhora com a radioterapia e com o uso de corticosteróides, geralmente não apresentam resposta tomográfica evidente. De modo geral, a radioterapia é paliativa em $66 \%$ a $75 \%$ dos casos e critérios tomográficos mostram melhora em $50 \%$ a $60 \%$ dos pacientes. Os tumores de menor tamanho respondem melhor e a presença de mais de três lesões confere prognóstico mais reservado ${ }^{4}$.

Ainda há outras questões controversas com relação à radioterapia, como uso de radiação profilática, reirradiação, uso de radioproterores e de medicações sinérgicas.

Em alguns serviços, utiliza-se a radioterapia cerebral profilática para os pacientes com diagnóstico de carcinoma pulmonar de pequenas células, que ainda não apresentaram metástases cerebrais, baseando-se no fato de que este tumor é radiossensível. Recentemente foram revisados 11 estudos realizados sobre essa questão e não foi demonstrado aumento da sobrevida, embora a maioria dos estudos descreva menor taxa de ocorrência de metástases para o sistema nervoso central ${ }^{8}$.

Apesar de muito freqüentemente os pacientes não sobreviverem tempo suficiente para a observação de manifestações clínicas tardias induzidas pela radioterapia, há relatos de neurotoxicidade tardia nos pacientes que alcançaram maior sobrevida. DeAngelis et al. ${ }^{25}$ relataram a ocorrência de complicações tardias induzidas pela radioterapia em pacientes que estavam aparentemente "curados" e que receberam doses de radioterapia variando entre 2.500 e 3.900 cGy. Entre as alterações descritas, há relatos de demência progressiva, ataxia e incontinência urinária.

$\mathrm{Na}$ maioria dos serviços, associam a radioterapia ao uso de corticosteróides. Estes, além de influen- ciarem na melhora clínica dos pacientes, também reduzem os efeitos colaterais da radioterapia, como náuseas e cefaléia, melhorando, assim, a qualidade de vida dos pacientes ${ }^{8}$.

Classicamente, admite-se que uma das grandes limitações da quimioterapia para o tratamento de metástases cerebrais é o fato de que as drogas comumente utilizadas não ultrapassariam a barreira hematoencefálica (BHE). Entretanto, há relatos de alterações na BHE nos tumores metastáticos e de penetração das drogas antineoplásicas nessas lesões ${ }^{26,27}$. Vários estudos prospectivos têm relatado resultados favoráveis de quimioterapia como tratamento primário para metástases cerebrais em pacientes com carcinomas de mama, adenocarcinoma e carcinoma pulmonar de pequenas células, neoplasia trofoblástica gestacional e de tumores germinativos ${ }^{26,27}$. Contudo, esses resultados ainda não foram observados em estudos randomizados. As evidências de potencial valor da quimioterapia incluem a medida da concentração das drogas antineoplásicas nas metástases, mostrando que níveis citotóxicos podem ser alcançados ${ }^{26,27}$. Também vale ressaltar que as metástases comumente surgem em fases avançadas do câncer, quando muitas vezes já há resistência aos antineoplásicos de primeira linha, de modo que a resposta esperada é sempre menor e, além disso, muitos tumores apresentam metástases para o sistema nervoso central, como o melanoma e o carcinoma renal, que são, geralmente, resistentes aos quimioterápicos de primeira linha ${ }^{27}$.

\section{Retratamento}

Nos pacientes com doença sistêmica controlada, a recorrência de metástases cerebrais previamente tratadas é relativamente comum ${ }^{8}$ e seu tratamento é especialmente difícil. Todos os dados referentes a essa questão são baseados em estudos retrospectivos. A reirradiação externa paliativa oferece uma sobrevida de 2 a 4 meses e, quando possível, a radiocirurgia proporciona melhor controle local da doença ${ }^{8}$. Em casos selecionados, uma nova cirurgia também pode ser útil ${ }^{28,29}$. A braquiterapia também pode ser uma opção, mas seu valor ainda não foi estabelecido ${ }^{8}$.

\section{Cuidados terminais}

A despeito de todos os avanços no tratamento das metástases cerebrais, o prognóstico desses pacientes permanece reservado, com sobrevida global em torno 
de 12 meses $^{8}$. Com relação aos cuidados terminais, o tratamento sintomático é fundamental, dando-se importância ao controle do edema cerebral e das crises epilépticas. Muitos pacientes têm doença disseminada sistemicamente e podem requerer analgesia para controle da dor, devendo-se ressaltar que os pacientes com metástases cerebrais são geralmente mais sensíveis aos opióides, podendo apresentar sonolência ou quadro confusional em decorrência da analgesia ${ }^{8}$.

\section{SUMMARY}

\section{Brain metastases}

Brain metastases are common in cancer patients. The frequency of this complication seems to be raising as a result of more aggressive treatment of cancer as well more accurate diagnosis by use of neuroimaging methods. The clinical relevance of the treatment becomes evident with the development of effective therapeutic approaches to control the primary tumor. The main purpose of the treatment is to reverse neurological deficits and to increase survival, besides improving quality-of-life. The authors review the clinical manifestations, diagnosis and the main treatment options for brain metastases.

\section{Keyw ords}

Intracerebral metastases, surgery, radiotherapy, clinical management.

\section{Referências}

1. Patchell AR. Metastatic brain tumors. In: Brain tumors in adults. Black PM, Wen PY (eds.). WB Saunnders Company, Philadelphia, 1995, pp. 95-9.

2. Nguyen LN, Maor MH, Oswald MJ. Brain metastases as the only manifestation of an undetecd primary tumor. Cancer, 83:2181-4, 1998.

3. Thapar K, Laws ER. Tumores Del Sistema Nervioso Central. In: Oncologia Clínica. Murphy GP, Lawrence W (eds.). American Cancer Society, Washington, 1995, pp. 424-62.

4. Posner JB. Intracranial metastases. In: Neurologic complications of cancer. Posner JB (ed.). FA Davis Company, Philadelphia, 1995, pp. 77-110,

5. Delattre JY, Krol G, Thaler HT, Posner JB. Distribuiton of Brain Metastases. Arch Neurol, 45:741-4, 1988.

6. Hwang TL, Close TP, Grego JM, Brannon WL, Gonzales $F$. Predilection of brain metastases in gray and white matter junction and vascular border zones. Cancer, 77:1551-5, 1996.

7. Yokoi K, Kamya N, Matsuguma H, Machida S, Hirose T et al. Detection of brain metastasis in potentially operable non-small cell lung cancer. A comparision of CT and MRI. Chest, 115:714-9, 1999.
8. Davey P. Brain metastases. Curr Probl Cancer, 23:5998, 1999.

9. Karlsson B, Ericson K, Kihlstrom L, Grane P. Tumor seeding following sterotactic biopsy of brain metastases. J Neurosurgery, 87:327-30, 1997.

10. Posner JB. Supportive care agents and their complications. In: Neurologic complications of cancer. Posner JB (ed.). FA Davis Company, Philadelphia, 1995, pp. 59-74.

11. Cohen N, Strauss R, Lew R, Silver D, Recht L. Should prophylactic anticonvulsivants be administred to patients with newly-diagnosed cerebral metastases? A retrospective analyses. J Cli Oncol, 6:1621-4, 1988.

12. Chumas GH, Condon B, Oluoch-Olunya D, Griffths S, Hadley $D$ et al. Early changes in peritumorous oedema and contralateral white matter after dexamethasone: a study using proton magnetic ressonance spectroscopy. J Neurol Neurosurg Psychiatry, 62:590-5, 1997.

13. Cockey GH, Amann ST, Reents SB, Lynch JW. SteevensJohnsons syndrome resulting from whole - Brain radiation and phenytoin. Am J Clin Oncol, 19:32-4, 1996.

14. Patchell RA, Tibbs PA, Walsh JW, Dempsey RJ, Maruyama $Y$ et al. A randomized trial of surgery in the treatment of single metastases to the brain. $\mathrm{N}$ Engl J Med, 322:494-500, 1990.

15. Vecht CJ, Haaxma - Reiche H, Noordijk EM, Padberg GW, Voormolen JHC et al. Treatment of single brain metastasis: radiotherapy alone or combined with neurosurgery? Ann Neurol, 33:583-90, 1993.

16. Cairncross JG, Kim JH, Posner JB. Radiaton therapy for brain metastases. Ann Neurol, 7:529-41, 1980.

17. DeAngelis LM, Mandell LR, Thaler HT, Kimmel DW, Galicich $\mathrm{JH}$ et al. The role of post-operative radiotherapy after ressection of single brain metastases. Neurosurgery, 24:798-805, 1989.

18. Sause WT, Crowley JJ, Morantz R, Rotmam M, Mowry PA et al. Solitary brain metastases: results of an RTOG / SWOG protocol evaluation surgery + RT versus $R T$ alone. Am J Clin Oncol, 13:427-32, 1990.

19. Laing RW, Warrington AP, Hines F, Graham JD, Brada M. Fractioned stereotactic external beam radiotherapy in the management of brain metastases. Eur $\mathrm{J}$ Cancer, 29:1387-91, 1993.

20. Lavine SD, Petrovich Z, Cohen - Gadol AA, Masri LS, Morton DL et al. Gamma knife radiosurgery for metastatic melanoma: an analysis of survival, outcome and complications. Neurosurgery, 44:59-66, 1999.

21. Cho KH, Hall WA, Gerbi BJ, Higgins PD, Bohen M et al. Patient selection criteria for the treatment of brain metastases with stereotactic radiosurgery. J Neurooncol, 40:73-86, 1998.

22. Alexander III E, Moriarty TM, Davis RB, Wen PY, Fine HA et al. Stereotactic radiosurgery for the definitive, noninvasive treatment of brain metastases. J Natl Cancer Inst, 87:34-40, 1995.

23. Bindal AK, Bindal RK, Hess KR, Shiv A, Hassenbush SJ et al. Surgery versus radiosurgery in the treatment of brain metastasis. J Neurosurg, 84:748-54, 1996.

24. Sneed PK, Lamborn KR, Forstner JM, McDermont MW, Chang $S$ et al. Radiosurgery for brain metastases: is whole brain radiotherapy necessary? Int J Radiation Oncology Biol Phys, 43:549-58, 1999.

25. DeAngelis LM, Delattre JY, Posner JB. Radiation-Induced dementia in patients cured of brain metastases. Neurology, 39:789-96, 1989 
26. Malacarne P, Santini A, Maestri A. Response of brain metastases from lung cancer to systemic chemotherapy with carboplatin and etoposide. Oncology, 53:210-3, 1996.

27. Siegers HP. Chemotherapy for brain metastases: recent developments and clinical considerations. Cancer Treat Ver, 17:63-76, 1990.

28. Arbit E, Wronski M, Burt M, Galicich JH. The treatment of patients with recurrent brain metastases. A retrospective analyses of 109 patients with non-small cell lung cancer. Cancer, 76:765-73, 1995.
29. Sundaresan N, Sachden VP, DiGiacinto GV, Hugghes JEO. Reoperation for brain metastases. J Clin Oncol, 6:1625-9, 1988.

\section{Endereço para correspondência:}

Adrialdo José Santos

Disciplina de Neurologia

Escola Paulista de Medicina - Unifesp

Rua Botucatu, 740

CEP 04023-900 - São Paulo, SP

E-mail: ajsnoncology@hotmail.com 